\title{
A Pneumococcal Carriage Study in Danish Pre-school Children before the Introduction of Pneumococcal Conjugate Vaccination
}

\author{
Zitta B. Harboe, Hans-Christian Slotved*, Helle B. Konradsen and Margit S. Kaltoft
}

Neisseria and Streptococcus Reference Center, Department of Microbiological Surveillance and Research, Statens Serum Institut, Copenhagen, Denmark

\begin{abstract}
We present data on pneumococcal carriage before the introduction of the heptavalent-pneumococcal conjugated vaccine (PCV7) in Denmark. In the pre-PCV7 period, the incidence of invasive pneumococcal disease (IPD) among children younger than 5 years was approximately 25 per 100.000 population, with the highest incidence rates observed in children younger than 2 years of age. The study included 437 children aged 12-72 months attending day care centres (DCC) and was conducted during 48 months. In total, $56 \%(n=247)$ of children were pneumococcal carriers with the highest prevalence in children aged 12-23 months (69\%), the proportion significantly declining with increasing age. PCV7 serotypes accounted for 33\%, PCV10 for 34\%, and PCV13 for $57 \%$ of all carried isolates. The proportion of serotypes included in the three conjugate vaccines was higher among IPD isolates compared to carrier isolates (range 35$90 \%$ ). We found that the frequency of carriage was high among Danish pre-school children attending DCC and serotypes were not frequently covered by PCV7 in the pre-PCV7 period.
\end{abstract}

Keywords: Children, nasopharyngeal carriage, Streptococcus pneumoniae, serotypes, vaccine.

\section{INTRODUCTION}

Infections caused by Streptococcus pneumoniae are major contributors to morbidity and the main cause of vaccine preventable deaths in children younger than 5 years of age worldwide [1, 2]. The range of infections caused by pneumococci is wide and often preceded by the asymptomatic carrier state in the nasopharynx, mainly in pre-school children [3]. From the nasopharynx, it may spread into the upper airway and middle ear, and cause mucosal, non-invasive disease, but can also invade sterile sites, such as blood and cerebrospinal fluid, causing severe invasive pneumococcal disease (IPD).

Factors such as age, ethnicity, immunosuppression including HIV-1 infection, socioeconomic factors like crowding, and temporal association to respiratory viral diseases widely affect the risk of IPD [4]. In particular, a high carriage prevalence has been associated with high incidences of pneumococcal disease, including IPD [5]. Attendance to day care centres (DCC) has been recognized as an important risk factor both with regard to acquisition of pneumococcal carriage and infections in pre-school children $[6,7]$. In Denmark, it is estimated that between $55-85 \%$ of children between 6 months and 5 years attend a DCC $[6,8]$.

The heptavalent-pneumococcal conjugate vaccine (PCV7, Pfizer Vaccines) has been shown to be highly effective in reducing IPD caused by vaccine serotypes in both vaccinated and non-vaccinated cohorts [9-12]. PCV7

*Address correspondence to this author at the Department of Microbiological Surveillance and Research, Statens Serum Institut, Artillerivej 5, DK-2300 Copenhagen, Denmark; Tel: +45 32688422; Fax: +45 32683865; E-mail: hcs@ssi.dk was introduced in the Danish childhood immunization programme in a $2+1$ schedule in October 2007; before that, PCV7 was only recommended for children younger than 2 years of age with high-risk conditions $[13,14]$. In the prePCV7 period, the incidence of IPD among children younger than 5 years in Denmark was approximately 25 per 100.000 population, with the highest incidence rates observed in children younger than 2 years of age [15]. The incidence of IPD nearly halved shortly after the introduction of the vaccine [16]. Pneumococcal conjugate vaccines of higher valence, including the 10 -valent pneumococcal non-capsulate $H$. influenzae protein-D conjugate vaccine (PhiD-CV; PCV10; GlaxoSmithKline Biologicals) and the 13-valent pneumococcal protein conjugate vaccine (PCV13; Pfizer Vaccines) provide broader serotype coverage $[11,15,17,18]$. PCV13 was started to be used in Denmark in April 2011 [28].

The aim of this study was to describe the serotype distribution of pneumococcal isolates carried in the nasopharynx of non-vaccinated infants attending DCCs in a local Danish district and in the pre-PCV7 period and to compare the proportion of serotypes included in three conjugate vaccines among carried and IPD isolates.

\section{MATERIALS AND METHODS}

\section{Study Setting and Design}

We performed a cross-sectional study in order to evaluate the prevalence of pneumococcal nasopharyngeal carriage among children aged 12-72 months attending 27 DCCs in the Municipality of Roskilde, Region Zealand during 1999-2000. Nurseries, kindergartens and combined DCCs (including both a nursery and a kindergarten in one 
institution) were eligible to be included in the study. In total, 437 children aged 12-72 months were included in the study.

Written informed consents were obtained from children's parents. The study was approved by The Regional Ethical Committee (register number KF 01-371/98 and KF 11094/00), the Department of Children and Culture in the Municipality of Roskilde, and the Danish Data Protection Agency (registry number 2001-41-1214). An analysis of a subgroup of this study population was previously presented by Auranen et al., [19] including only 3 DCCs out of the 27 DCCs.

\section{Sampling and Identification of Pneumococcal Isolates}

Sampling was performed as previously described by Kaltoft et al., [17]. Briefly, a trained person collected a deep nasopharyngeal sample using a calcium alginate-tipped aluminum wire swab (Calgi swab type 1, Puritan, Hardwood Products Company LP, USA). The swab was inserted into a test tube containing $1.5 \mathrm{ml}$ serum broth and kept at $5^{\circ} \mathrm{C}$ until it was plated in the laboratory within 3-5 hours after sampling. Pneumococcal isolates were identified and serotyped at the Neisseria and Streptococcus Reference Centre (NSR), Statens Serum Institut as previously described $[16,20]$.

The serotypes included in PCV7 are 4, 6B, 9V, 14, 18C, 19F, and 23F. PCV10 includes PCV7 serotypes plus serotypes 1, 5, and 7F; and PCV13 includes PCV10 serotypes plus serotypes 3,6A, and 19A.

\section{Data Analysis}

Categorical data were compared using chi-square test, $p$ values $\leq 0.05$ were considered significant. The proportion of children who were pneumococcal carriers by age group was estimated and expressed as carrier rates (CR). Also, we calculated the proportion of PCV7, PCV10 and PCV13 serotypes among IPD isolates by age group using data from the nationwide laboratory surveillance system [21].

\section{RESULTS}

At least one pneumococcal isolate from the nasopharyngeal sample was found in $56.5 \%$ of all children $(n=247), 54 \%$ of the 247 children were males. A second serotype was found in $9 \%$ of children $(n=22)$. The highest prevalence of pneumococcal carriage, 69\% $(n=27)$, was found among the youngest children (age 12-23 months) and tended to decline each year dropping to a carriage rate of $53.8 \%$ at five years of age and to $46.2 \%$ at six years of age.

The serotypes included in PCV7 accounted for 33\%, 34\% were PCV10 serotypes, and 57\% were PCV13 serotypes among all carried isolates, with the highest serotype coverage of the vaccines among the youngest children. Serotypes found in children aged either $12-23$ or $24-35$ months were mainly those included as additional serotypes contained in PCV13. In children from the age group 36-47 months and older, non-vaccine serotypes were more frequently observed (Fig. 1). The serotype coverage of the three conjugate vaccines was higher in IPD compared to carrier isolates (range 35-90\%), differing significantly between the groups $(p=0.001, p=<0.001, \quad p=0.006$ respectively) (Fig. 2). Also, the proportion of carriage isolates covered by PCV7 and PCV13 decreased significantly with an increasing age ( $p=0.04$ and $p=0.001$ respectively).

\section{DISCUSSION}

To our knowledge, this is the largest report documenting the prevalence of pneumococcal serotypes in nasopharyngeal carriage in pre-school children before the introduction of PCV7 into the Danish Childhood Immunization Programme. We found a high prevalence of pneumococcal carriage, highest in children aged 12-23 months, where nearly $70 \%$ were carriers. Serotypes included in PCV7 were not commonly represented, only in about $30 \%$ of carried isolates.

We found that both the carrier rates and the serotype distribution of carried isolates varied markedly with age. The high prevalence of carriage among the youngest children coincides with the observation that the incidence of IPD is highest in this age group [8]. Paediatric serotypes/groups (6, 14,19 and 23) were found frequently both in carriers and as a cause of IPD in the youngest children in the pre-PCV7 period. These serotypes/groups are all represented in PCV7, explaining the higher coverage of the PCV7 among IPD cases in children $<23$ months, for whom vaccination is attempted (Fig. 2). The predominance of serotypes included in PCV7 declined significantly with increasing age, both among carriers and IPD cases, in accordance with findings reported from other populations [2,9]. Serotype 5 is not a frequent cause of paediatric IPD in Denmark, while serotypes 1 and 7F become more important in IPD in older children and adults $[8,16]$; all these factors may determine a relatively low coverage of PCV10 among carried isolates in our study population. The higher serotype coverage of PCV13 was mainly related to the presence of serotype $6 \mathrm{~A}$ in both carriage and in IPD (Fig. 2). These findings are comparable to those reported from a number of carriage studies from different geographic locations [7, 11, 22, 23]. Also, the analysis of risk factors for the carriage of vaccine serotypes showed that age, gender, size of DCC, type of DCC, and month of sampling were not found to be significantly associated with an increased risk of carrying an isolate belonging to a serotype included in PCV7, PCV10 or PCV13 (data not shown).

The pneumococcal capsular polysaccharide has been described as a major determinant of the duration of carriage, relative invasiveness, disease presentation and mortality related to IPD $[13,20]$. It also appears that the biochemical structure of the capsule determines the success of a given serotype in nasopharyngeal carriage [24]. In spite of differences in study design and population, a number of studies have identified a few serotypes as having the greatest invasive potential. Similar to our findings, a study from Gambia reported that serotypes/groups 1, 12 and 14 were more frequently found in patients with severe pneumococcal disease than in carriers, while serogroups 19 and 23 were often isolated from healthy controls [5]. In a study from India, serotypes/groups 1, 5, 7, 12 were prevalent among invasive isolates, but not among carriers [25]. Also, a study 

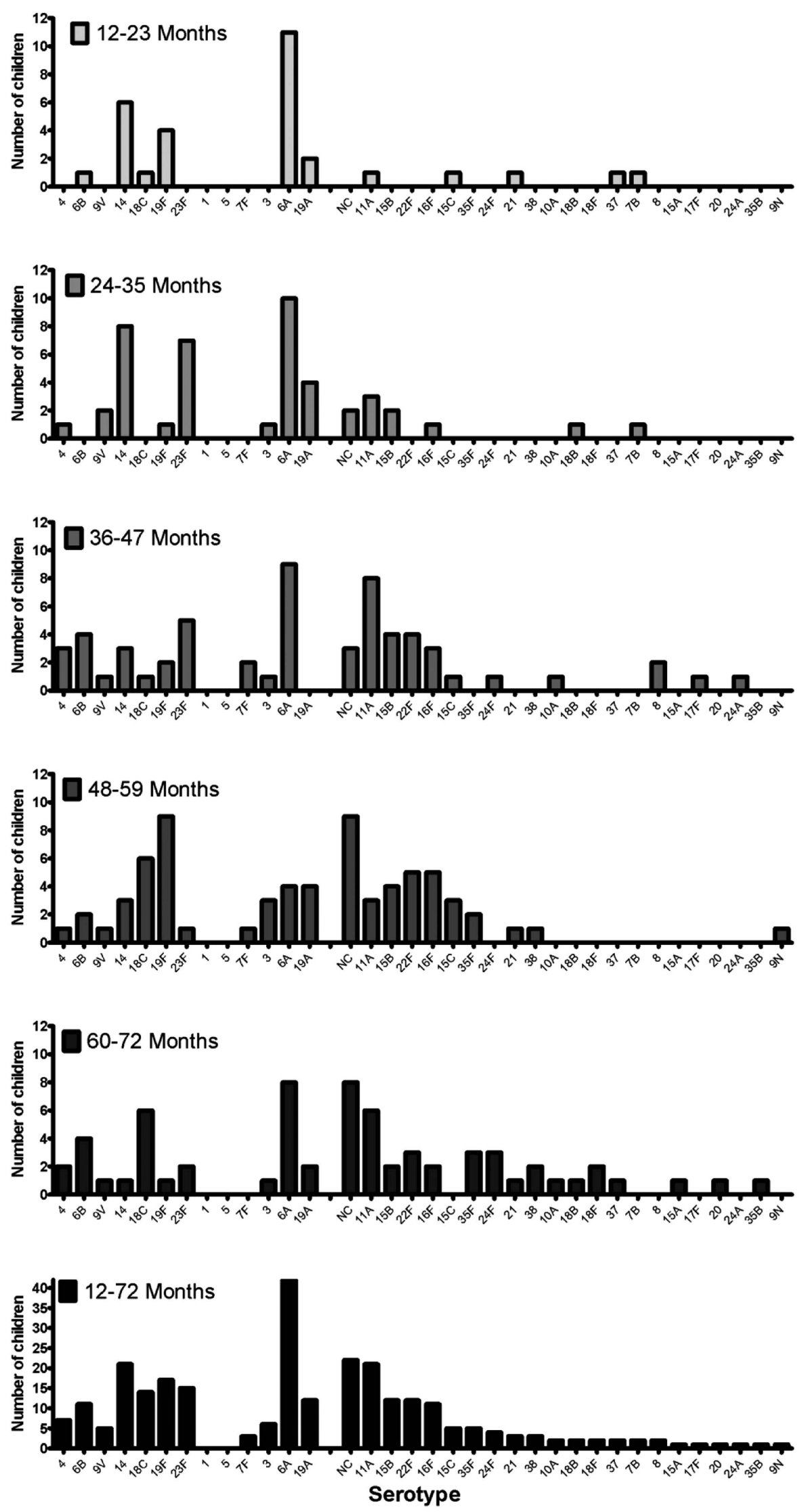

Fig. (1). Prevalence of pneumococcal serotypes represented among 269 isolates colonizing 247 pre-school children according to age group. Among all carriers $(\mathrm{n}=247), 22$ children carried 2 isolates. 

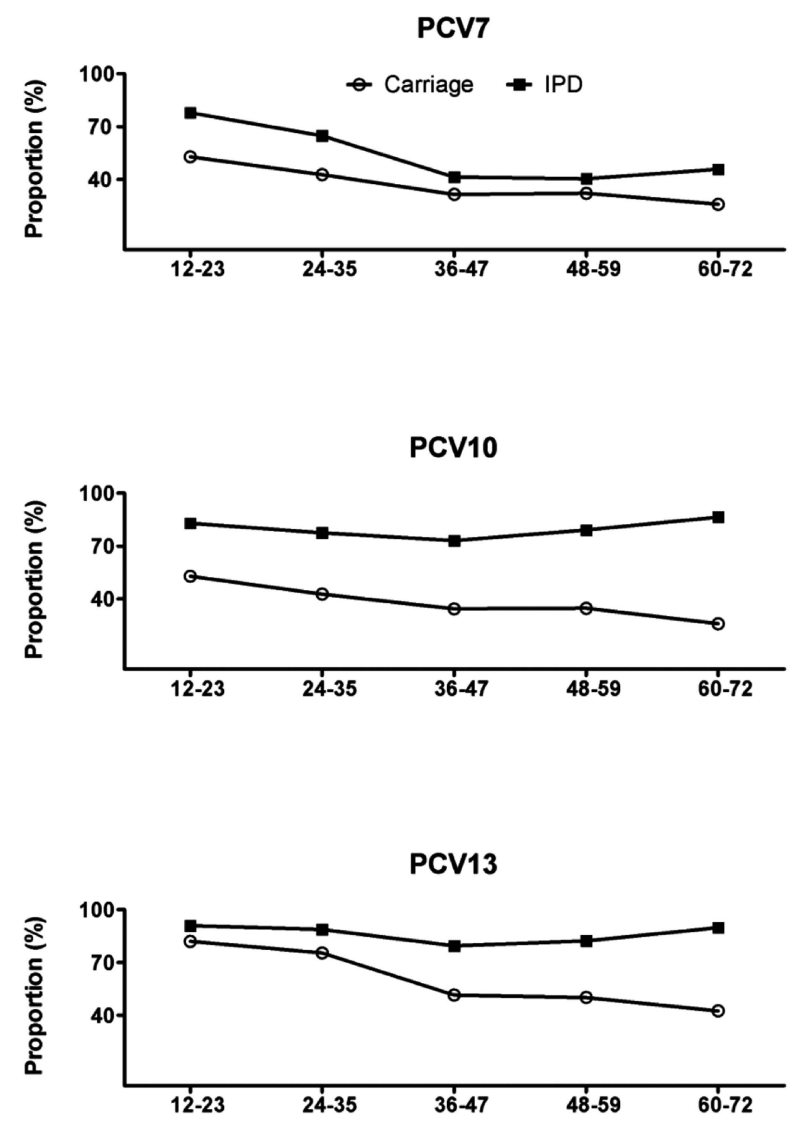

Fig. (2). Proportion of invasive $(n=700)$ and carried $(n=269)$ pneumococcal isolates covered by three different pneumococcal conjugate vaccines* as a function of age in months.

*The serotypes included in the seven valent pneumococcal conjugate vaccine (PCV7) are serotypes 4, 6B, 9V, 14, 18C, 19F, and 23F. The 10-valent pneumococcal non-capsulate $\mathrm{H}$. influenzae protein-D conjugate vaccine (PCV10) includes PCV7 serotypes plus serotypes 1, 5 and $7 \mathrm{~F}$, and the 13-valent pneumococcal protein conjugate vaccine (PCV13) includes PCV10 serotypes plus serotypes 3, 6A, and 19A.

from Papua New Guinea [26] reported that serogroups 11, 15 and 22 were of low invasiveness, similar to what we found in our study. A study from Oxford reported that PCV7 serotypes showed to be associated with high invasiveness [27]. The consistency between these reports supports the fact that the invasiveness of capsular serotypes is a serotypespecific characteristic. However, even if capsular serotype is a factor that determines the invasiveness of a particular strain, the study population may determine the prevalence of host-related factors (such as genetic predisposition, the prevalence of co-morbid conditions and socioeconomic factors), which influence the invasiveness of serotypes.

In conclusion, pneumococcal colonization in children attending DCCs is frequent, highest in the youngest infants, and represents a heterogeneous population of pneumococcal serotypes. Studies investigating the nasopharyngeal pneumococcal carriage in the coming years would be of major interest in order to evaluate the dynamics of the pneumococcal population in the post-PCV7 period.

\section{CONFLICT OF INTEREST}

This research was partially supported by a grant from Leo Pharma Nordic and Statens Serum Institut. An analysis of a subgroup of this study population was previously presented by Auranen et al., [19] including only 3 out of the 27 DCCs. No other conflicts of interest are declared.

\section{ACKNOWLEDGEMENTS}

We acknowledge the Danish Departments of Clinical Microbiology for submitting invasive pneumococcal isolates for national surveillance and the staff performing the serotyping of the isolates at the NSR. We also thank Azra Kurbazic, MSc, $\mathrm{PhD}$, for assistance in the statistical analysis.

\section{REFERENCES}

[1] Brugger SD, Hathaway LJ, Mühlemann K. Detection of Streptococcus pneumoniae strain cocolonization in the nasopharynx. $\mathrm{J}$ Clin Microbiol 2009; 47: 1750-6.

[2] Dagan R, Givon-Lavi N, Zamir O, et al. Reduction of nasopharyngeal carriage of Streptococcus pneumoniae after administration of a 9 valent pneumococcal conjugate vaccine to toddlers attending day care centers. J Infect Dis 2002; 185: 927-36.

[3] O'Brien KL, Wolfson LJ, Watt JP, et al. Burden of disease caused by Streptococcus pneumoniae in children younger than 5 years: global estimates. Lancet 2009; 374: 893-902.

[4] Hausdorff WP, Feikin DR, Klugman KP. Epidemiological differences among pneumococcal serotypes. Lancet Infect Dis 2005; 5: 83-93.

[5] Evans LN, O'Dempsey TJ, Baldeh I, et al. Nasopharyngeal carriage of pneumococci in Gambian children and in their families. Pediatr Infect Dis J 1996; 15: 866-71.

[6] Nielsen A, Lie HR, Keiding L, Madsen M. Børn og børnefamiliers levevilkår i Danmark. In Børns sundhed i Danmark. Dansk institut for klinisk epidemiologi, Copenhagen 1998; pp. 261-265..

[7] Syrjanen RK, Kilpi TM, Kaijalainen TH, Herva EE, Takala AK. Nasopharyngeal carriage of Streptococcus pneumoniae in 
Finnish children younger than 2 years old. J Infect Dis 2001; 184: 451-9.

[8] Kaltoft MS, Zeuthen N, Konradsen HB. Epidemiology of invasive pneumococcal infections in children aged 0-6 years in Denmark: a 19-year nationwide surveillance study. Acta Paediatr Suppl 2000; 89: 3-10.

[9] Hausdorff WP, Bryant J, Paradiso PR, Siber GR. Which pneumococcal serogroups cause the most invasive disease: implications for conjugate vaccine formulation and use, part I. Clin Infect Dis 2000; 30: 100-21.

[10] Whitney CG, Farley MM, Hadler J, et al. Active Bacterial Core Surveillance Program of the Emerging Infections Program Network. Increasing prevalence of multidrug-resistant Streptococcus pneumoniae in the United States. N Engl J Med 2000; 343: 1917-24.

[11] Hussain M, Melegaro A, Pebody RG, et al. A longitudinal household study of Streptococcus pneumoniae nasopharyngeal carriage in a UK setting. Epidemiol Infect 2005; 133: 891-8.

[12] Hsu HE, Shutt KA, Moore MR, et al. Effect of pneumococcal conjugate vaccine on pneumococcal meningitis. $\mathrm{N}$ Engl J Med 2009; 360: 244-56.

[13] Harboe ZB, Thomsen RW, Riis A, et al. Pneumococcal serotypes and mortality following invasive pneumococcal disease: a population-based cohort study. PLoS Med 2009; 26: 6:e1000081.

[14] Hjuler T, Wohlfahrt J, Simonsen J, et al. Perinatal and crowdingrelated risk factors for invasive pneumococcal disease in infants and young children: a population-based case-control study. Clin Infect Dis 2007; 44: 1051-6.

[15] Harboe ZB, Valentiner-Branth P, Benfield TL, et al. Estimated effect of pneumococcal conjugate vaccination on invasive pneumococcal disease and associated mortality, Denmark 20002005. Vaccine 2008; 26: 3765-71

[16] Harboe ZB, Valentiner-Branth P, Benfield TL, et al. Early effectiveness of heptavalent conjugate pneumococcal vaccination on invasive pneumococcal disease after the introduction in the danish childhood immunization programme. Vaccine 2010; 28: 2642-7.

[17] Kaltoft MS, Sørensen SUB, Slotved H-C, Konradsen HB. An easy method for detection of nasopharyngeal carriage of multiple Streptococcus pneumoniae serotypes. J Microbiol Methods 2008; 75: $540-4$

[18] Sá-Leão R, Nunes S, Brito-Avô A, et al. High rates of transmission of and colonization by Streptococcus pneumoniae and Haemophilus influenzae within a day care center revealed in a longitudinal study. J Clin Microbiol 2008; 46: 225-34.

[19] Auranen K, Mehtälä J, Tanskanen AS, Kaltoft M. Between-strain competition in acquisition and clearance of pneumococcal carriage-epidemiologic evidence from a longitudinal study of day-care children. Am J Epidemiol 2010; 171: 169-76.

[20] Sleeman KL, Griffiths D, Shackley F, et al. Capsular serotypespecific attack rates and duration of carriage of Streptococcus pneumoniae in a population of children. J Infect Dis 2006; 194: 682-8.

[21] Harboe ZB, Benfield TL, Valentiner-Branth P, et al. Temporal trends in invasive pneumococcal disease and pneumococcal serotypes over 7 decades. Clin Infect Dis 2010; 50: 329-37.

[22] Millar EV, O'Brien KL, Zell ER, Bronsdon MA, Reid R, Santosham M. Nasopharyngeal carriage of Streptococcus pneumoniae in Navajo and White Mountain Apache children before the introduction of pneumococcal conjugate vaccine. Pediatr Infect Dis 2009; 28: 711-6.

[23] Syrogiannopoulos GA, Katopodis GD, Grivea IN, Beratis NG. Antimicrobial Use and Serotype Distribution of Nasopharyngeal Streptococcus pneumoniae Isolates Recovered from Greek Children Younger than 2 Years Old. Clin Infect Dis 2002; 35: 1174-82.

[24] Weinberger DM, Trzciński K, Lu YJ, et al. Pneumococcal capsular polysaccharide structure predicts serotype prevalence. PLoS Pathog 2009; 5: e1000476.

[25] Jebaraj R, Cherian T, Raghupathy $\mathrm{P}$, et al. Nasopharyngeal colonization of infants in southern India with Streptococcus pneumoniae. Epidemiol Infect 1999; 123: 383-8.

[26] Smith T, Lehmann D, Montgomery J, Gratten M, Riley ID, Alpers MP. Acquisition and invasiveness of different serotypes of Streptococcus pneumoniae in young children. Epidemiol Infect 1993; 111: 27-39.

[27] Brueggemann AB, Griffiths DT, Meats E, Peto T, Crook DW, Spratt BG. Clonal relationships between invasive and carriage Streptococcus pneumoniae and serotype- and clone-specific differences in invasive disease potential. J Infect Dis 2003; 187: 1424-32.

[28] EPI-NYT, 2011, uge 19. www.ssi.dk • epinyt@ssi.dk • ISSN: 1396-8599, Statens Serum Institut, Denmark.

Received: December 01, 2011

Revised: February 20, 2012

Accepted: February 27, 2012

(C) Harboe et al.; Licensee Bentham Open.

This is an open access article licensed under the terms of the Creative Commons Attribution Non-Commercial License (http://creativecommons.org/licenses/ by-nc/3.0/) which permits unrestricted, non-commercial use, distribution and reproduction in any medium, provided the work is properly cited. 